\title{
EL PAPEL DE LA MORFOLOGÍA EN EL RECONOCIMIENTO LÉXICO, ESPAÑA
}

THE ROLE OF LEXICAL MORPHOLOGY IN THE RECOGNITION, SPAIN

\author{
Miguel Lázaro Lópe.VillaseñoR ${ }^{1}$ \\ Universidad de Castilla la Mancha. Dpto. de Psicología, España \\ (RECIBIDO El 22/09/2010, ACEPTADO EL 22/11/2010)
}

\begin{abstract}
RESUMEN
En el léxico se almacenan y procesan un enorme número de palabras. Este almacenamiento supone un coste cognitivo que no parece asumible por las capacidades del cerebro si no fuera por la especial organización del léxico, que ordena las entradas de modo que economiza el coste de este gran caudal de palabras. En la investigación de dicha organización léxica, destaca desde hace tres décadas el estudio del papel que desempeña la morfología. Diversas investigaciones, tanto con personas con lesión cerebral como sin lesión, han mostrado que la morfología efectivamente cumple un papel muy importante para el reconocimiento léxico. Este papel, sin embargo, no es conocido en profundidad. En este trabajo realizamos una revisión en profundidad de las principales investigaciones al respecto y discutimos los resultados a la luz de los diversos modelos teóricos propuestos.
\end{abstract}

Palabras clave: Acceso al léxico, morfología, reconocimiento léxico.

\begin{abstract}
In the lexicon there are stored and processed a huge number of words. Taking into account the limited capacities of the brain, it is only possible this store because of the economical structure of the lexicon. On the research of the lexical structure stands out from three decades the study of the role played by morphology. A number of investigations, with and without brain damaged subjects, have shown that morphology plays an important role on the lexical access, although its role is still not very well know. In this study we make a deep revision of the main researches concerning the role of morphology, and discuss the results on the light of the different models proposed.
\end{abstract}

Keywords: Lexical access, lexical processing, morphology. 


\section{INTRODUCCIÓN}

Uno de los mayores avances de la Psicolingüística en las últimas décadas ha sido el comenzar con el estudio sistemático del papel de la morfología en el reconocimiento léxico. Taft y Forster (1975) fueron los primeros autores en trabajar experimentalmente en este ámbito, abriendo con aquella publicación clásica un campo de investigación que sería, con el tiempo, muy productivo. En el trabajo de 1975, Taft y Forster concluyeron que el reconocimiento léxico visual de palabras complejas (formadas por más de un morfema) se veía precedido por una etapa de procesamiento en la que dichas palabras se segmentaban en unidades morfológicas. Ésta es, exactamente, la propuesta contraria a la que realizó Butterworth (1983), quien investigó también el reconocimiento léxico visual manipulando claves morfológicas y encontró que las palabras no habían sido descompuestas en unidades morfológicas como paso previo a su reconocimiento, es decir, que la (des)composición morfológica de las palabras no había sido relevante en el reconocimiento. De este hallazgo, Butterworth concluyó que las palabras complejas se reconocen sin mediación de procesamiento morfológico alguno. Las propuestas de Taft y Forster (1975) y Butterworth (1983) han constituido los extremos sobre los que se puede concebir el papel de la morfología en el reconocimiento léxico; o bien tiene un papel crucial o bien no tiene ningún papel. Entre ambas propuestas se ubican la mayor parte de los trabajos que se han ido publicando en los últimos años. No obstante, existen autores y cierta evidencia que sustenta las conclusiones de los modelos clásicos.

\section{Modelos conexionistas}

Entre la multitud de campos de investigación que se han visto enriquecidos con el desarrollo de los modelos conexionistas se encuentra el del reconocimiento léxico. Los modelos conexionistas han estudiado la morfología y su relevancia dentro de la organización léxica y han defendido siempre la hipótesis de que la morfología no tiene entidad por sí misma en el reconocimiento léxico, sino en relación con otros niveles de análisis del lenguaje. De este modo, para los autores conexionistas, los resultados experimentales encontrados que habitualmente se interpretan como un efecto de carácter morfológico, serían evidencia de la interacción semántica y fonológica que la composición morfológica conlleva (e.g. Devlin, Jamison, Matthews y Gonnerman, 2004; Seidenberg, 1993; Seidenberg y Gonnerman, 2000). Bybee (1985) expone este hecho muy sencilla e inteligentemente, sostiene que si dos términos se relacionan semántica y fonológicamente, entonces también se relacionan morfológicamente.

\section{Argumentos teóricos de procesamiento morfológico}

Cuando Taft y Forster (1975) realizaron su investigación, conocían las propuestas y estudios de los lingüistas sobre la morfología, pero no contaban con datos experimentales previos que pudieran hacerles formar una hipótesis clara acerca de lo que podrían encontrar con experimentos de reconocimiento visual en los que se manipulaba la morfología. De este modo, los únicos estudios disponibles eran plenamente teóricos. En este nivel teórico existe también la dicotomía entre los que niegan el nivel morfológico 
y los que lo defienden, aunque existen hechos lingüísticos que parecen apoyar, a nuestro modo de ver, un procesamiento morfológico de las palabras complejas. Podemos destacar tres importantes fenómenos: El primero se refiere a la regularidad de los procesos morfológicos, especialmente en lo que respecta a la morfología flexiva. Taft y Forster estudiaron inicialmente la morfología flexiva, conocedores de que la morfología derivativa es menos regular, presentando más excepciones. En efecto, si los procesos morfológicos son regulares, o al menos bastante regulares, entonces el sistema puede computar eficazmente la información morfológica, puesto que es predecible. En el caso de que la morfología fuera plenamente irregular, carente de reglas, el sistema no podría valerse eficazmente de la morfología para realizar el reconocimiento léxico y tendría que realizarse un reconocimiento global de cada una de las palabras. Hankamer (1989), basado en los resultados de su estudio computaciona.cognitivo, ha puesto de manifiesto la imposibilidad de tal reconocimiento. A la misma conclusión llega Moreno (2000), aunque desde un punto de vista plenamente teórico. El segundo fenómeno se ciñe a la finitud del inventario de morfemas. Como sabemos, de la concatenación de un número limitado -aunque extenso- de lexemas y un número limitado -y no muy extenso- de morfemas gramaticales, resulta la totalidad de las palabras de nuestro léxico, que son de extraordinaria magnitud numérica. Por lo tanto, el cerebro maneja una cantidad enorme de palabras, pero éstas se forman a partir de un número mucho menor de elementos. El principio de economía (lingüística), axioma de la ciencia, sustenta todo aquello que supone un ahorro en cualquier término, y en este caso se expresa un ahorro en términos computacionales cuando se indica que procesar los morfemas previamente a las palabras es más económico que procesar las palabras sin previo reconocimiento de los morfemas. El tercer fenómeno, relacionado con el punto anterior, consiste en que el número de palabras de una lengua, de la familia indoeuropea al menos, son más usualmente palabras complejas que simples (Booij, 2005; Rey-Debove, 1984), es decir, aquellas que están compuestas por dos o más morfemas son más numerosas que aquellas otras compuestas por un único morfema como "sol", "luna", "agua", etc. Si fuera al contrario, es decir, si hubiera más palabras simples que complejas, entonces el beneficio del procesamiento morfológico sería escaso, pero no es así.

\section{Indicios experimentales de procesamiento morfológico}

El número de investigaciones experimentales que observa datos coherentes con un procesamiento morfológico de las palabras complejas es elevado. Además, es muy relevante que esta evidencia se refiera también a la independencia del procesamiento morfológico con respecto del semántico y fonológico, es decir, que contrariamente a las predicciones y propuestas ya expuestas de los modelos conexionistas, contamos con datos empíricos que avalan la independencia del nivel morfológico. Longtin y Meunier (2005) observaron que, efectivamente, el procesamiento morfológico no es producto del solapamiento semántico y fonológico. En el primero de los tres experimentos que realizaron, Longtin y Meunier (2005) observaron que las pseudopalabras formadas por lexemas y morfemas ambos existentes facilitaban la lectura de sus bases en igual medida que auténticas palabras derivadas, es decir, que las palabras que comparten la base léxica con la palabra objetivo. Por ejemplo, la pseudopalabra "cantista", compuesta por un lexema "cant" y un morfema 
"ista", facilitaba la lectura de la base "canta" en igual medida que una palabra realmente existente como "cantante" en comparación con un grupo control (el grupo control estaba representado por palabras sin relación semántica ni ortográfica con las bases propuestas). Estos resultados podían explicarse de tres formas distintas y relacionadas, por separado, con cada uno de los niveles de análisis gramatical. De acuerdo con una hipótesis ortográfica, la semejanza del patrón ortográfico existente entre las pseudopalabras y palabras con respecto a sus bases -semejanza que no se presenta en el grupo contro. podía explicar que tanto las palabras como las pseudopalabras hubieran facilitado la lectura de las palabras objetivo. Por su parte, de acuerdo con una hipótesis semántica, la clave podría encontrarse en la transparencia semántica de las pseudopalabras. Dado que tanto las palabras como las pseudopalabras utilizadas fueron semánticamente interpretables o transparentes, existía entre las palabras y las pseudopalabras una correspondencia semántica con sus bases. Esta correspondencia podría explicar, de acuerdo con la hipótesis semántica, la facilitación de la lectura de las cadenas objetivo. Por último, también una hipótesis morfológica podía explicar los resultados; si hubiera existido un procesamiento morfológico, tanto las palabras como las pseudopalabras -todas ellas complejas- podrían haber facilitado el procesamiento de sus bases. Este procesamiento morfológico habría separado los anticipadores en sus componentes morfológicos y la base habría sido activada a través de su relación morfológica con los estímulos anticipadores. Al producirse esta segmentación morfológica, tanto en el caso de las palabras como en el de las pseudopalabras, el efecto facilitador se habría presentado en ambos tipos de estímulo. Como estos resultados tienen una interpretación distinta según las distintas hipótesis, Longtin y Meunier (2005) diseñaron dos experimentos más para determinar el alcance de los resultados de este primer experimento. En el primero de ellos examinaron la hipótesis ortográfica. Observaron en los resultados que el solapamiento ortográfico mostraba un efecto significativo, pero distinto a cuando este solapamiento se producía dentro de una familia morfológica, es decir, aunque se asociaba un efecto significativo al solapamiento ortográfico/fonológico entre palabras no relacionadas, éste era de distinta naturaleza a cuando se producía dentro de una familia morfológica. De este modo se sustentaba el que la morfología hubiera tenido un papel independiente del solapamiento ortográfico en el primer experimento. En otro experimento posterior examinaron la hipótesis semántica. Para ello crearon palabras complejas transparentes y pseudopalabras en las que el morfema y el lexema fueran legales pero que pertenecieran a categorías gramaticales distintas, por ejemplo un morfema deverbal adjuntado a un lexema nominal: "car.able". La lógica del experimento era que si se encontraba facilitación en la lectura de las bases de las palabras complejas tanto usando como anticipador las palabras transparentes como usando como anticipador las pseudopalabras también complejas y opacas, el resultado sería prueba de que habría existido descomposición morfológica. En el caso de que se encontrara facilitación para el caso de las palabras transparentes y no para el de las pseudopalabras opacas podría defenderse que los resultados del primer experimento, y del resto, se debían al solapamiento de significados. Los resultados, finalmente, mostraron que tanto las palabras complejas como las pseudopalabras opacas facilitaron la lectura de las bases objetivo. Por todo ello, Longtin y Meunier (2005) concluyeron que el léxico es sensible a la composición morfológica de las palabras y que el efecto asociado al procesamiento morfológico no se debe ni al solapamiento de significados ni al solapamiento ortográfico de las familias morfológicas -ni a la suma de ambos efectos. 
A esta conclusión habían llegado anteriormente Rastle, Davis, Marsle.Wilson y Tyler (2000) o Feldman (2000), que realizaron estudios en los que la relación entre anticipadores y palabras objetivo se manipularon de modo que entre ellos hubiera distintas relaciones. En los resultados encuentran efectos asociados al solapamiento ortográfico y semántico, pero observan también que existe un efecto morfológico que no puede ser explicado en función de los otros efectos, de modo que se sustenta su estatuto independiente.

Acerca de los modelos teóricos que explican el procesamiento morfológico, cabe destacarse aquellos denominados híbridos por tomar en cuenta e integrar algunas de las propuestas de los modelos más extremos, aquellos que niegan la descomposición morfológica (Butterworth, 1983) y aquellos que proponen una descomposición obligatoria para todos los estímulos (Taft y Forster (1975). El modelo de Caramazza, Laudanna y Romani (1988) denominado AAM; Augmented Adressed Morphology; Morfología Ampliada Accesible por el Contenido o el de Schreuder y Baayen (1995), basado, como el AAM, en el modelo de Ruta Dual (Coltheart, 1978) son quizá los modelos que más eco han tenido. El modelo de Caramazza et al. (1988) tuvo su origen en los resultados que mostraron que en tareas de decisión léxica, aquellas pseudopalabras que estaban compuestas por lexemas y morfemas derivativos, ambos existentes pero con cuya concatenación no se generaban verdaderas palabras, eran más difícil de rechazar que aquellas otras que, aún siendo fonotácticamente legales, no estaban constituidas de este modo. Para Caramazza (1988) estos resultados evidencian una sensibilidad del sistema hacia los componentes morfológicos constitutivos de las palabras. De no ser así, justifican, no podrían explicarse los resultados citados. Para el modelo AAM, aquellos resultados que muestran la no existencia de procesamiento morfológico durante el reconocimiento léxico se explican por la frecuencia de los estímulos empleados, de modo que, como el modelo de Ruta Dual en el que se basa, se propone que las palabras de alta frecuencia no requieren descomposición morfológica, mientras que las de baja frecuencia sí la requieren. La frecuencia de palabra es una variable ciertamente relevante para el procesamiento morfológico, pero existe otra variable también sustancial. Schreuder y Baayen (1995) o Aronoff y Fudeman (2005), en su propuesta teórica consideran que las palabras semánticamente opacas no requieren descomposición morfológica, mientras que las transparentes sí. De este modo, "gatillo", que es una palabra opaca porque tiene asociado un significado distinto al de "gato pequeño", que es lo que debería significar de acuerdo a sus morfemas constitutivos, no podría ser procesada en base a sus componentes morfológicos porque, si así fuera, se extraería de la concatenación de sus unidades un significado que no se correspondería con el que realmente le corresponde; "percutor". No obstante, Schreuder, Burani y Baayen (2003) encontraron datos opuestos a esta propuesta. Observaron que las palabras, independientemente de su estatuto semántico, eran descompuestas en sus componentes morfológicos. Efectivamente, observaron en una tarea de decisión léxica en la que el anticipador se presentó durante un tiempo de 500 min. que las bases, tanto de las palabras transparentes como de las opacas, habían sido segmentadas y que además se había producido una activación semántica de las mismas. La activación de las bases de forma independiente al estatuto semántico de las palabras demuestra que todas las palabras habían sido no solo descompuestas, sino además que se había producido una activación semántica de los morfemas. En la actualidad existe evidencia que confirma esta etapa obligatoria y temprana de segmentación morfológica (e.g., Lavric, Clapp y 
Rastle, 2007; Leinonen, Brattico, Järvenpää y Krause, 2008; Meunier y Longtin, 2007; Marsle.Wilson, Bozic y Randall, 2008).

\section{Curso temporal del procesamiento morfológico}

El curso temporal del procesamiento morfológico es una fuente importante de discusión dentro del grupo de investigadores que consideran que la morfología, efectivamente, se procesa durante el reconocimiento léxico. Por ejemplo, los modelos híbridos citados de Caramazza et al. (1988) y de Schreuder y Baayen (1995), pese a que sustentan un procesamiento léxico genuinamente morfológico, no se ponen de acuerdo en cuanto al modo de entender las relaciones de este procesamiento con el de otras variables y niveles. Pero además de ello, los modelos que comprenden un análisis morfológico se enfrentan de forma teórica por cómo conciben el curso temporal del procesamiento morfológico. En este sentido, existen dos grandes propuestas, las propuestas supraléxica y subléxica.

\section{Propuesta supraléxica}

La propuesta supraléxica se refiere, en oposición a la subléxica, al hecho de que primero se accedan o se procesen las palabras y posteriormente los elementos morfológicos que componen las palabras, es decir, que el procesamiento de las palabras complejas preceda al procesamiento de los componentes morfológicos que conforman dicha palabra. El modelo supraléxico, aunque considera que existe un procesamiento a nivel de palabra, no niega que pueda existir un procesamiento a nivel morfémico, aunque siempre posterior al que corresponde a la forma completa de la palabra.

Giraudo y Grainger (2000) observaron en tareas de decisión léxica en las que se anticipaban palabras objetivo con anticipadores morfológicamente complejos, que las latencias de respuestas disminuían si los anticipadores eran de alta frecuencia de uso en comparación con cuando eran de baja frecuencia. De este modo, las palabras objetivo a las que se anticipaba información frecuente resultaban facilitadas con respecto a aquellas otras a las que se anticipaba información poco frecuente. Estos resultados suponen para Giraudo y Grainger (2000) apoyo al modelo supraléxico, porque demuestran que el efecto morfológico generado depende del procesamiento de la palabra completa, es decir, de su forma no segmentada. Si ocurriera al revés, es decir, un procesamiento subléxico, no se esperarían diferencias en las latencias entre los anticipadores con alta y baja frecuencia por cuanto en ambos casos se activarían en primer lugar los morfemas, que en ambos tipos de palabras, de alta y baja frecuencia, eran los mismos y sólo posteriormente las palabras y sus significados.

Giraudo y Grainger (2001) observaron también evidencia a favor del modelo supraléxico. En el estudio de Giraudo y Grainger (2001) se realizó una serie experimental con tareas de decisión léxica y anticipadores enmascarados. En su primer experimento presentaron tres tipos distintos de condiciones que expresan distintas relaciones entre el anticipador y el objetivo. En el grupo control no existía relación alguna entre ambos estímulos. En otro grupo el anticipador presentaba el lexema en común con la palabra objetivo, si bien sólo a nivel ortográfico puesto que no era realmente el mismo lexema, es decir, se trataba de 
un pseudolexema. Un ejemplo de esto son las palabras "rato" y "rata". Ambas palabras presentan un lexema ortográficamente idéntico, pero no se trata realmente del mismo lexema. El último grupo de palabras creado mantenía entre el anticipador y la palabra objetivo un pseudomorfema en común, es decir, el mismo tipo de relación que en el ejemplo anterior salvo que en este caso con respecto al morfema derivativo y no al lexema. Giraudo y Grainger (2001) encontraron con esta manipulación que las distintas palabras objetivo de su trabajo no mostraron latencias de respuesta significativamente distintas entre sí. En el mismo trabajo publicado, pero en un segundo experimento, Giraudo y Grainger (2001) observaron que si evitaban los pseudolexemas y los peudomorfemas y utilizaban como anticipadores palabras complejas compuestas por el mismo lexema que la palabra objetivo más un morfema existente, entonces sí variaban significativamente las latencias de respuesta de las palabras objetivo con respecto a las del grupo control. Los resultados conjuntos del primer y segundo experimento demuestran para Giraudo y Grainger (2001) que el procesamiento llevado a cabo por los sujetos es de carácter supraléxico, porque de lo contrario las latencias de respuesta no deberían haber sido significativamente distintas en las condiciones en las que se utilizó un anticipador y una palabra objetivo que compartían un pseudolexema y un anticipador y una palabra objetivo que compartían un mismo lexema. En efecto, si bajo la hipótesis subléxica el sujeto extrajera primero, y muy tempranamente, los constituyentes morfológicos de la palabra sin consideración del resto de información, entonces, al ser el lexema y el pseudolexema idénticos en la forma, el sujeto no podría haberlos juzgado como distintos. Por ello, ambos tipos de anticipadores habrían tenido que producir una activación similar, mostrándose en ambos grupos de palabras una facilitación idéntica. Las propuestas del modelo supraléxico, por el contrario, sí permitirían explicar los resultados observados. Para el modelo supraléxico no existe una descomposición morfológica previa al análisis del estímulo léxico en su conjunto, motivo por el que el reconocimiento de palabras en las que se hubiera anticipado un pseudolexema o un mismo lexema sería esencialmente distinto y, por tanto, se explicaría el encontrar resultados distintos entre ambos grupos de estímulos.

Pero la evidencia experimental no apoya exclusivamente un procesamiento de tipo supraléxico. En favor del modelo subléxico existe también evidencia empírica relevante. Carreiras, Perdomo, y Mesenguer (2005) analizaron distintas evidencias publicadas sobre procesamiento subléxico y supraléxico y concluyeron que existen resultados que sustentan sólidamente tanto un tipo de procesamiento como otro, por lo que no consideran factible defender un modelo frente a otro hasta que comprendamos los motivos de la discrepencia. En esta línea, Diependaele, Sandra y Grainger (2005) afirman que "hasta donde conocemos, no existe ningún modelo de procesamiento morfológico que pueda explicar todos los resultados presentados" (pp. 102).

\section{Modelo subléxico}

El procesamiento subléxico se refiere, en oposición al procesamiento supraléxico, al hecho de que primero se accedan o procesen los elementos morfológicos de las palabras y posteriormente su forma completa, es decir, que el procesamiento de los elementos morfológicos de las palabras complejas preceda al procesamiento de éstas en su forma completa. 
Existe abundante evidencia relevante a favor del procesamiento subléxico. Fabre, Meunier y Hoen (2007), encuentran, utilizando palabras complejas como anticipadores y palabras simples como objetivos, que la facilitación producida por el anticipador era independiente de la frecuencia del mismo. Este resultado es el contrario al anteriormente citado de Giraudo y Grainger (2000) y así lo reconocen en la discusión de este trabajo Fabre et al. (2007). Para intentar comprender esta divergencia, Fabre (2007) analiza la metodología comparativamente entre ambos trabajos. Consideran tras este análisis que el tiempo de exposición del anticipador pudiera ser la razón de tan dispares resultados, pero no profundizan o examinan suficientemente a nuestro juicio esta hipótesis. En su experimento se expuso a los sujetos a un anticipador de $47 \mathrm{~min}$. mientras que en el experimento de Giraudo y Grainger (2000) se trataba de un anticipador de 57 min. Nuestra opinión es que estos 10 min de diferencia difícilmente podrían hacer variar los resultados de forma tan substancial dado que el anticipador de $57 \mathrm{~min}$. debe considerarse, como el de $47 \mathrm{~min}$., breve. En este sentido Grainger, Colé y Segui (1991) y Rastle, Davis, Marsle.Wilson y Tyler (2000) consideran breves incluso anticipadores de $72 \mathrm{~min}$. y $64 \mathrm{~min}$., respectivamente.

Para defender un procesamiento subléxico en la lectura y reconocimiento de palabras, existe un argumento importante y sólido, se trata de los resultados antes citados de Caramazza et al. (1988). Estos autores demostraron que es más difícil rechazar en tareas de decisión léxica aquellas pseudopalabras que contienen morfemas o lexemas, o ambos, que aquellas otras que no están formadas por este tipo de constituyentes. Con otras variables controladas, este hallazgo supone un respaldo para el modelo subléxico y no para un modelo supraléxico, que no podría dar cuenta del porqué de estos resultados. El modelo subléxico sí sería eficaz en la explicación. Propondría que primero se accedería a la representación de los morfemas y posteriormente al significado a través de la forma completa de la palabra. Una vez que tanto las palabras como las pseudopalabras comparten constituyentes, la decisión se hace más compleja al resultar activados distintos tipos de objetivos. Longtin y Meunier (2005) basándose en los resultados de sus experimentos también defienden este procesamiento de carácter subléxico. Encontraron que las pseudopalabras compuestas por lexema + sufijo producían, utilizadas como anticipadores, un efecto facilitador sobre la lectura de palabras objetivo similar al de palabras complejas. Sin embargo, si la pseudopalabra no respetaba la forma de lexema + morfema, entonces no se producía facilitación. Longtin y Meunier (2005) y Meunier y Longtin (2007) defienden que existe un proceso de descomposición morfológica temprano que se aplica a todas y cada una de las entradas. Este procesamiento es necesariamente temprano dado que no se muestra afectado por el carácter semántico de la entrada, procesamiento éste que sería posterior a la descomposición morfológica.

\section{Morfología y daño cerebral}

Existen numerosos estudios que, con pacientes afásicos, han encontrado deficiencias en el procesamiento morfológico de los sujetos. Estos problemas, cuando no ocurren junto a otros de tipo semántico y fonológico, o cuando lo hacen de forma autónoma e independiente, se pueden considerar también indicios de procesamiento morfológico. Los trastornos del lenguaje han sido una fuente inmensa de información para los procesos que subyacen al 
lenguaje (Sereno y Rayner, 2003). La investigación con pacientes que presentan lesiones cerebrales que derivan en trastornos lingüísticos es por tanto una fuente importante de información sobre el papel de la morfología y su estatuto (Debazer y Semenza, 1998; Ullman y Gopnik, 1999). Un ejemplo relevante lo constituye la investigación de Aitchison (2004). Este autor trabaja con pacientes afásicos y obtiene resultados importantes desde el punto de vista teórico. Aitchinson (2004) llevó a cabo una serie de estudios con un único paciente y observó que este paciente tenía problemas en la formación de plurales de baja frecuencia, pero no en la formación de plurales de alta frecuencia. Este paciente, además, no tenía dificultades con la derivación, sino únicamente con la flexión. Este tipo de pacientes, según Aitchison, suponen un apoyo empírico a aquellas propuestas que defienden que la flexión y la derivación no son tan solo dos procesos morfológicos distintos a nivel teórico, sino que lo son, también, a nivel cognitivo. En efecto, si existen pacientes que tienen dificultades en los mecanismos flexivos y no en los derivativos, esta hipótesis de distinción entre ambos procesos morfológicos cobraría fuerza, más aún si encontramos disociaciones dobles, como ocurre con Laudanna, Badecker y Caramazza (1992). Laudanna et al. han presentado varios casos en los que los pacientes manifiestan problemas con la derivación pero no con la flexión y viceversa.

Algunos estudios con pacientes afásicos han sido, pues, sustanciales para discutir o defender determinadas propuestas teóricas referentes a la morfología. Las disociaciones dobles que se pueden encontrar en los trabajos citados respecto a los trastornos en la formación morfológica de las palabras son pruebas indirectas de que el sistema es sensible a la composición morfológica de las palabras y que ésta es relevante para el procesamiento de las palabras. Por tanto, empíricamente también se ha mostrado que la morfología tiene un papel relevante en el procesamiento léxico.

\section{CONCLUSIONES}

Existe una importante evidencia, tanto teórica como experimental, que sustenta un papel relevante de la morfología en el reconocimiento léxico. Aunque existen autores que niegan esta evidencia, los datos globales sí apuntan hacia un procesamiento morfológico de las entradas léxicas complejas. Existen aparentes contradicciones en los resultados observados que dificultan el entendimiento de cómo y cuándo se produce la segmentación y posterior concatenación morfológica -procesamiento morfológico-, pero esta discusión no entorpece la conclusión final de un procesamiento morfológico cierto, así como tampoco dificulta por ejemplo la aplicación de ejercicios clínicos que, centrados en el procesamiento morfológico, ayuden a los pacientes afásicos o con trastornos lectoescritores (Arnbak y Elbro, 2000; Lázaro, 2010).

\section{REFERENCIAS BIBLIOGRÁFICAS}

1. Aitchison, J. (2004). Speech percetion and production. En G. Booij, C. Lehman y J. Mugdan (Eds.), Morphology: An international handbook of inflection and word formation (pp. 345-358). Berlin: De Gruyter. 
El PAPEL DE LA MORFOLOGÍA EN EL RECONOCIMIENTO LÉXICO

2. Arnbak, E., \& Elbro, C. (2000). The effects of morphological awareness training on the reading and spelling skills of young dyslexics. Scandinavian Journal of Educational Research, 44, 229-251.

3. Booig, G. (2002). The morphology of Dutch. Oxford:Oxford University Press.

4. Butterworth, B. (1983). Lexical representations. En: B. Butterworth (Ed.), Language production (pp. 257-294). Londres: Academic Press.

5. Bybee, J. (1985). Morphology: A study between meaning and form. Amsterdam/ Phladelphia: John Benjamins.

6. Caramazza, A., Laudanna, A., y Romani, C. (1988). Lexical access and inflectional morphology. Cognition, 28, 297-332.

7. Carlisle, J., y Katz, C. (2006). Effects of word and morpheme familiarity on reading a derived word. Reading and writing. An Interdisciplinary Journal,17, 669-693.

8. Carreiras, M., Perdomo, A., y Mesenguer, E. (2005). Are stem homographs and orthographic neighbors processed differently during silent reading? Language and Cognitive Processes, 20 (1/2), 317-339.

9. Coltheart, M. (1978). Lexical access in simple reading tasks. G. Underwood (Ed.), Strategies of information processing (pp. 151-216). London: Academic Press.

10. Debazer, M., y Semenza, C. (1998). The processing of compound words: A study in aphasia. Brain and Language, 61, 54-62.

11. Devlin, J.T., Jamison, H.L.; Matthews, P.M. y Gonnerman, L.M. (2004). Morphology and the internal structure of words. Proceedings of the National Academy of Sciences of the United States of America, 101 (41), 14984-14988.

12. Diependaele, K.; Sandra, D., y Grainger, J. (2005). Masked cros.modal morphological priming: Unravelling morph.orthographic and morph.semantic influences in early word recognition. Language and Cognitive Processes, 20, (1/2), 75-114.

13. Fabre, D., Meunier, F., y Hoen, M. (2007). Role of surface frequency on morphological family organization. En S. Vosniadou., D. Kayser., y A. Protopapas (Eds.), Proceedings of the European Cognitive Science Conference. Delphi: Tylor and Francis.

14. Feldman, B. (2000). Are morphological effects dintinguishable from the effects of shared meaning and shared form? Journal of experimental psychology. Learning, memory, and cognition, 26, 1431-1444.

15. Giraudo, H., y Grainger, J. (2000). Effects of prime word frequency and cumulative root frequency in masked morphological priming. Language and Cognitive Processes, 15(4/5), 421-444.

16. Giraudo, H., y Grainger, J. (2001). Priming complex words: Evidence for supralexical representation of morphology. Psychonomic Bulletin and Review, 8, 127-131. 
17. Hankamer, J. (1989). Morphological parsing and the lexicon. En W. Marsle. Wilson (Ed.), Lexical representations and process (pp. 392-408). Massachussets: MIT Press.

18. Laudanna, A., Badecker, W., y Caramazza, A. (1992). Processing inflectional and derivational morphology. Journal of Memory and Language, 31, 333-348.

19. Lavric, A., Clapp, A., y Rastle, K. (2007). ERP evidence of morphological analysis from orthography: a masked priming study. Journal of Cognitive Neuroscience, 19, 866-877.

20. Lázaro, M. (2010). El papel del procesamiento morfológico en el reconocimiento léxico: Implicaciones de cara a una intervención logopédica en trastornos afásicos. Boletín de la Revista de Logopedia, Foniatría y Audiología, 10, 8-11.

21. Leinonen, A., Brattico, P., Järvenpää, M., y Krause, C. (2008). Even.related potential (ERP) responses to violations of inflectional and derivational rules of Finnish. Brain Research, 1218, 181-193.

22. Longtin, C. M., y Meunier, F. (2005). Morphological decomposition in early visual word processing. Journal of Memory and Language, 53, 26-41.

23. Marsle.Wilson, W., Komisarjevsky, L., Waksler, R., y Older, L. (1994). Morphology and meaning in the English mental lexicon. Psychological Review, 101(1), 3-33.

24. Meunier, F., y Longtin, .M. (2007). Morphological decomposition and semantic integration in word processing. Journal of Memory and Language, 56, 457-471.

25. Moreno, J.C. (2000). Curso universitario de lingüística general. Madrid: Síntesis.

26. Rastle, K., Davis, M., Marsle.Wilson, W. y Tyler, L. (2000). Morphological and semantic effects in visual word recognition: A time course study. Language and Cognitive Processes, 15(4/5), 507-537.

27. Re.Debove, J. (1984). Le domaine de la morphologie lexicale. Cahiers de Lexicologie, 45, 3-19.

28. Schreuder, R., y Baayen, R.H. (1995). Modeling morphological processing. En B. Feldman (Ed.), Morphological aspects of of language processing, pp. 131-154 Hillsdale, New Yersey: Erlbaum.

29. Schreuder, R., Burani, C., y Baayen, R.H. (2003). Parsing and semantic opacity. E. Assink \& D. Sandra (Eds.), Morphology and the mental lexicon (pp. 14-49). Dordrecht: Kluwer.

30. Seidenberg, M. (1993). Connectionist models and cognitive theory. Psychological Science, 4, 22.235.

31. Seidenberg, M., y Gonnerman, L. (2000). Explaining derivational morphology as the convergence of codes. Trends in Cognitive Sciences, 4(9), 353-361.

32. Sereno, S., y Rayner, K. (2003). Measuring word recognition in reading: eye movements and event related potentials. Trends in Cognitive Sciences, 7(11), 489493. 
EL PAPEL DE LA MORFOLOGÍA EN EL RECONOCIMIENTO LÉXICO

33. Taft, M., y Forster, K. (1975). Lexical storage and retrieval of polymorphemic and pollysillabic words. Journal of Verbal Learning and Verbal Behavior, 15 (6), 607-620.

34. Ullman, M., y Gopnik, M. (1999). Inflectional morphology in a family with inherited specific language impairment. Applied Psycholinguistics, 20, 51-117. 